Bryn Mawr College

Scholarship, Research, and Creative Work at Bryn Mawr College

1986

\title{
A Partial Integration Formula for Product Integrals of Unbounded Operator-Valued Functions
}

Rhonda J. Hughes

Bryn Mawr College, rhughes@brynmawr.edu

Let us know how access to this document benefits you.

Follow this and additional works at: http://repository.brynmawr.edu/math_pubs

Part of the Mathematics Commons

\section{Custom Citation}

Hughes, Rhonda J. "A Partial Integration Formula for Product Integrals of Unbounded Operator-Valued Functions." Proc. Amer. Math. Soc. 96 (1986): 455-461.

This paper is posted at Scholarship, Research, and Creative Work at Bryn Mawr College. http://repository.brynmawr.edu/math_pubs/5

For more information, please contact repository@brynmawr.edu. 


\title{
A PARTIAL INTEGRATION FORMULA FOR PRODUCT INTEGRALS OF UNBOUNDED OPERATOR-VALUED FUNCTIONS
}

\author{
RHONDA J. HUGHES ${ }^{1}$ \\ ABSTRACr. The partial integration formula for product integrals

$$
\prod_{y}^{x} e^{(A(s)+B(s)) d s}=\prod_{y}^{x} e^{A(s) d s} \prod_{y}^{x} \exp \left(\left(\prod_{s}^{y} e^{A(u) d u} B(s) \prod_{y}^{s} e^{A(u) d u}\right) d s\right),
$$ \\ of which the Trotter product formula is a consequence, is established for a wide class \\ of unbounded operator-valued functions $A(s), B(s)$.
}

In $[6,7]$, Masani discusses the relationship between the "partial integration formula" for product integrals and the Trotter product formula, deriving the latter from the former for bounded operator-valued functions. He also asks whether an analogous relationship (from which Trotter's formula for unbounded operators would follow) can be established for unbounded operator-valued functions. In this note we address this question, providing conditions under which a partial integration formula for unbounded operators is valid, and exploring its implications for the Trotter product formula. We now describe our main results.

Let $X$ be a Banach space, and let $\mathscr{C}(X)$ denote the set of closed linear operators on $X$. Set

$$
\mathscr{K}=\{A(\cdot):[0, t] \rightarrow \mathscr{C}(X)
$$

(i) for each $s \in[0, t], A(s)$ is the infinitesimal generator of a contraction semigroup,

(ii) $0 \in \bigcap_{s \in[0, t]} \rho(A(s))$,

(iii) the $A(s)$ have a common dense domain $D$, and

(iv) $A(s)$ is strongly $C^{1}$ on $D$.

It was first shown by Kato (cf. [5], also [2, Theorem 12, 3, Theorem 6.1, p. 112, 9, p. 430]) that for $A(\cdot) \in \mathscr{K}$, the product integral

$$
U_{A}(t, 0)=\prod_{0}^{t} e^{A(s) d s}=s-\lim _{n \rightarrow \infty} \prod_{k=1}^{n} \exp \left(A\left(\frac{(k-1) t}{n}\right) \frac{t}{n}\right)
$$

Received by the editors October 23, 1984 and, in revised form, April 1, 1985.

1980 Mathematics Subject Classification. Primary 47D05, 47D10; Secondary 34G10.

${ }^{1}$ This work was partially supported by a grant from the National Science Foundation. 
exists; in fact, the product integral $U_{A}(x, y)$ converges for all $x, y \in[0, t], y \leqslant x$, $U_{A}(x, y) D \subset D$, and

$$
\frac{d}{d x} U_{A}(x, y) \phi=A(x) U_{A}(x, y) \phi, U_{A}(y, y) \phi=\phi,
$$

for all $\phi \in D, 0 \leqslant y \leqslant x$. Moreover, $U_{A}(x, y)$ is a contraction on $X$ for $0 \leqslant y \leqslant x$, and the propagator identity $U_{A}(x, r) U_{A}(r, y)=U_{A}(x, y)$ holds for $0 \leqslant y \leqslant r \leqslant x$.

RemarK. Assumptions (i)-(iv) in the definition of $\mathscr{K}$ imply the existence of a convergent product integral for $A(s)-I$; multiplying that product integral by $e^{x-y}$ then gives rise to the product integral of $A(s)$ (cf. [2, p. 344]). In fact, in (ii), 0 may be replaced by any $\lambda$ for which $A(s)-\lambda I$ satisfies the remaining assumptions. We then prove:

Theorem 1. Let $A(\cdot) \in \mathscr{K}$, and for each $s \in[0, t]$, let $B(s)$ be the infinitesimal generator of a contraction semigroup, such that $C(s) \equiv A(s)+B(s)$ also belongs to $\mathscr{K}$, with common dense domain $D$. Then

$$
U_{C}(t, 0)=s-\lim _{n \rightarrow \infty} \prod_{k=1}^{n} U_{A}(k t / n,(k-1) t / n) \exp \left(B\left(\frac{(k-1) t}{n}\right) \frac{t}{n}\right) .
$$

THEOREM 2. In addition to the hypotheses of Theorem 1, assume that for each $s$, $D(A(s)) \subset D(B(s))$, and $A(s)$ is the infinitesimal generator of a strongly continuous group of contractions. Then the partial integration formula

$$
\prod_{0}^{t} e^{(A(s)+B(s)) d s}=\prod_{0}^{t} e^{A(u) d u} \prod_{0}^{t} \exp \left(\left(\prod_{s}^{0} e^{A(u) d u} B(s) \prod_{0}^{s} e^{A(u) d u}\right) d s\right)
$$

holds.

As corollaries, we obtain (*) if, in addition to the hypotheses of Theorem 1, all the operators in question are skew-adjoint (Corollary 1), or if $A(s) \in \mathscr{K}$, and $B(s)$ is bounded, dissipative, and strongly $C^{1}$ (Corollary 2 ).

To clarify the ideas, let us first consider the case where $A(\cdot):[a, b] \rightarrow B(X)$ is continuous in the strong operator topology. Then the (strong) product integral

$$
\prod_{y}^{x} e^{A(s) d s} \equiv s-\lim _{n \rightarrow \infty} \prod_{k=1}^{n} e^{A\left(\bar{s}_{k}\right) \Delta s_{k}}
$$

exists for all $x, y \in[a, b]$; here $y=s_{0}<s_{1}<\cdots<s_{n}=x, \Delta s_{k}=s_{k}-s_{k-1}$, and $\bar{s}_{k} \in\left[s_{k-1}, s_{k}\right]$ (cf. [2, Theorem 1, Corollary 2]). Now, assume that, in additon, $B(\cdot)$ : $[a, b] \rightarrow B(X)$ is strongly continuous. Then the partial integration formula (or sum rule; cf. [2, Theorem 8,3 , Theorem 5.5]) holds:

$$
\prod_{y}^{x} e^{(A(s)+B(s)) d s}=\prod_{y}^{x} e^{A(s) d s} \prod_{y}^{x} \exp \left(\left(\prod_{s}^{y} e^{A(u) d u} B(s) \prod_{y}^{s} e^{A(u) d u}\right) d s\right) .
$$

Note. Part of the conclusion of (1) is that the product integral of $\prod_{s}^{y} e^{A(u) d u} B(s) \prod_{y}^{s} e^{A(u) d u}$ exists; here $\prod_{v}^{s} e^{A(u) d u}$ is invertible, and $\left(\prod_{v}^{s} e^{A(u) d u}\right)^{-1}=$ $\prod_{s}^{y} e^{A(u) d u}$.

The Trotter product formula,

$$
e^{t(A+B)}=\lim _{n \rightarrow \infty}\left(e^{t / n A} e^{t / n B}\right)^{n},
$$


then follows easily from (1), via the intermediate formula

$$
\prod_{y}^{x} e^{(A(s)+B(s)) d s}=\operatorname{sim}_{n \rightarrow \infty} \prod_{k=1}^{n} e^{A\left(\bar{s}_{k}\right) \Delta s_{k}} e^{B\left(\bar{s}_{k}\right) \Delta s_{k}},
$$

and the trivial fact that $B^{-1} e^{A} B=e^{B^{-1} A B}$, for bounded operators $A$ and $B$, with $B^{-1}$ bounded. (To obtain (2), reverse the roles of $A(s)$ and $B(s)$ in (1).)

To some extent, Masani's question about unbounded operators is answered by earlier work of Faris (cf. [4]), who establishes a version of (2) for (unbounded) contraction semigroup generators $A(s)$ and $B(s)$, provided some technical conditions are satisfied.

Faris proves

$$
\prod_{0}^{t} e^{(A(s)+B(s)) d s}=s-\lim _{n \rightarrow \infty} \prod_{k=1}^{n} \exp \left(A\left(\frac{(k-1) t}{n}\right) \frac{t}{n}\right) \exp \left(B\left(\frac{(k-1) t}{n}\right) \frac{t}{n}\right),
$$

provided basically that for each $s \in[0, t], C(s) \equiv A(s)+B(s)$ is closed, and possesses a convergent product integral. Although our ultimate goal is to establish a partial integration formula from which, for example, $\left(2^{\prime}\right)$ would follow, it is expeditious to work backwards, generalizing Faris's formula first. More precisely, suppose that instead of $\left(2^{\prime}\right)$, we could establish the following (which, in fact, corresponds to Corollary 2.6 in [7]): (let $x=0, y=t$ )

$$
\prod_{0}^{t} e^{(A(s)+B(s)) d s}=s-\lim _{n \rightarrow \infty} \prod_{k=1}^{n}\left(\prod_{(k-1) t / n}^{k t / n} e^{A(u) d u}\right) \exp \left(B\left(\frac{(k-1) t}{n}\right) \frac{t}{n}\right) .
$$

Proceeding formally, the right-hand side of (3) may be written (with $s_{k}=k t / n$ ) as

$$
\begin{aligned}
& s-\lim _{n \rightarrow \infty} \prod_{0}^{t} e^{A(s) d s} \prod_{k=1}^{n}\left(\prod_{s_{k-1}}^{0} e^{A(u) d u} e^{B\left(s_{k-1}\right) \Delta s_{k}} \prod_{0}^{s_{k-1}} e^{A(u) d u}\right) \\
& \quad=\sum_{n \rightarrow \infty}-\lim _{0} \prod^{A(s) d s} \prod_{k=1}^{n} \exp \left(\prod_{s_{k-1}}^{0} e^{A(u) d u} B\left(s_{k-1}\right) \prod_{0}^{s_{k-1}} e^{A(u) d u} \Delta s_{k}\right) \\
& \quad=\prod_{0}^{t} e^{A(u) d u} \prod_{0}^{t} \exp \left(\prod_{s}^{0} e^{A(u) d u} B(s) \prod_{0}^{s} e^{A(u) d u} d s\right),
\end{aligned}
$$

which is (1). Therefore, following the program suggested above, we shall first establish (3); then, in cases where the above formal calculation (4) may be made rigorous, we will show that Masani's question has an affirmative answer. Now, for the details.

Proof OF THEOREM 1. Following the standard proofs of the existence of product integrals for elements of $\mathscr{K}$ (cf. [2, Theorem 12, and 9, p. 430]), we introduce the family of operators

$$
W_{A}(t, s)=A(t) U_{A}(t, s) A(s)^{-1} .
$$

(Remark. Although the product integrals in [2] and [5] are defined differently, they are in fact identical by virtue of their being solutions of the same initial value problem.) Using Dollard and Friedman's approach (cf. [2, p. 350]) we see that

$$
W_{A}(t, s)=\sum_{n=0}^{\infty} U_{A}^{(n)}(t, s), \quad \text { where } U_{A}^{(0)}(t, s)=U_{A}(t, s),
$$


and

$$
\begin{aligned}
U_{A}^{(n)}(t, s)=\int_{s}^{t} U_{A}(t, u)(-C(u)) U_{A}^{(n-1)}(u, a) d u, & \\
& \text { where } C(u)=A(u) \cdot\left(A(u)^{-1}\right)^{\prime},
\end{aligned}
$$

which turns out to be strongly continuous for $A(\cdot) \in \mathscr{K}$ (cf. [2, Theorem 12, Corollary 1]). Moreover, $W_{A}(t, s)$ is jointly strongly continuous, and (trivially) satisfies the relation $A(t) U_{A}(t, s)=W_{A}(t, s) A(s)$. Now, we proceed as in [4], setting for $1 \leqslant k \leqslant n$, and $h=t / n$,

Then for $\phi \in D$,

$$
\begin{aligned}
P_{k} & =U_{A}(k h,(k-1) h), \\
Q_{k} & =B^{((k-1) h) h}, \\
R_{k} & =U_{C}(k h,(k-1) h), \quad \text { and } \\
S_{n} & =\prod_{k=1}^{n} P_{k} Q_{k}-U_{C}(t, 0) .
\end{aligned}
$$

$$
\begin{aligned}
\left\|S_{n} \phi\right\|= & \left\|\left(\prod_{k=1}^{n} P_{k} Q_{k}-\prod_{k=1}^{n} R_{k}\right) \phi\right\| \\
\leqslant & \sum_{j=1}^{n} \sum_{k=j+1}^{n}\left\|P_{k} Q_{k}\left[P_{j} Q_{j}-R_{j}\right] R((j-1) h, 0) \phi\right\| \\
\leqslant & n \sup _{0 \leqslant s \leqslant t}\left\|\left[U_{A}(s+h, s) e^{B(s) \cdot h}-U_{C}(s+h, s)\right] U_{C}(s, 0) \phi\right\| \\
\leqslant & \left.t \sup _{\substack{0 \leqslant s \leqslant t \\
\psi \in \mathscr{K}}}\left\|\left(\frac{1}{h}\left[U_{A}(s+h, s)-I\right]-A(s)\right) \psi\right\|\right) \\
& +\sup _{\substack{0 \leqslant s \leqslant t \\
\psi \in \mathscr{X}}}\left\|\left(U_{A}(s+h, s) \cdot \frac{1}{h}\left[e^{B(s) h}-I\right]-B(s)\right) \psi\right\| \\
& +\sup _{\substack{0 \leqslant s \leqslant t \\
\psi \in \mathscr{K}}}\left\|\left(\frac{1}{h}\left[U_{C}(s+h, s)-I\right]-C(s)\right) \psi\right\|,
\end{aligned}
$$

where $\mathscr{K}=\left\{U_{C}(s, 0) \phi, 0 \leqslant s \leqslant t\right\}$, so that $\mathscr{K} \subset D$. We must show that each of these terms converges to zero, as $n \rightarrow \infty$. For the first term on the right, we have, for $\phi \in D$,

$$
\begin{aligned}
\left\|\frac{1}{h}\left[U_{A}(s+h, s)-I\right] \phi-A(s) \phi\right\| & =\left\|\frac{1}{h} \int_{s}^{s+h} A(u) U_{A}(u, s) \phi-A(s) \phi d u\right\| \\
& =\left\|\frac{1}{h} \int_{s}^{s+h} W_{A}(u, s) A(s) \phi-A(s) \phi d u\right\| \\
& \leqslant \sup _{s \leqslant u \leqslant s+h}\left\|\left[W_{A}(u, s)-I\right] A(s) \phi\right\| .
\end{aligned}
$$

Clearly for fixed $s$, the latter approaches zero as $h \rightarrow 0$, for $W_{A}(s, s)=I$ and $W_{A}(u, s)$ is strongly continuous. However, the convergence is uniform in $s$; indeed, for $\phi \in X,\left\|W_{A}(s+h, s) \phi-\phi\right\| \rightarrow 0$ as $h \rightarrow 0$, uniformly in $s$, by compactness of 
$[0, t]$. Moreover,

$$
\begin{aligned}
\left\|W_{A}(s+h, s) \phi\right\| & =\left\|U_{A}(s+h, s) \phi+\sum_{n=1}^{\infty} U_{A}^{(n)}(s+h, s) \phi\right\| \\
& \leqslant\left\|U_{A}(s+h, s) \phi\right\|+\sum_{n=1}^{\infty} \frac{\int_{s}^{s+h}\|C(u)\| d u}{n !} \\
& \leqslant\|\phi\|+\sum_{n=1}^{\infty} \frac{M \cdot h\|\phi\|}{n !},
\end{aligned}
$$

where $\|C(u)\| \leqslant M$, by strong continuity, so $\left\|W_{A}(s+h, s)\right\|$ are uniformly bounded for, say $0<h \leqslant 1$. Since $\{A(s) \phi: 0 \leqslant s \leqslant t\}$ is compact in $X$, the convergence in (6) is uniform in $s$ (cf. [4, Lemma 1]). That the convergence is uniform on $\mathscr{K}$ follows precisely as in Faris's proof, by using the uniform boundedness theorem for the operators $(1 / h)\left[U_{A}(s+h, s)-I\right]$ and $A(s)$ on $B(D, X)$, where $D$ is regarded as a Banach space, with the graph norm of any $C(s)$ (cf. [4, Proposition 1]).

The convergence of the remaining terms in (5) is proved by observing that for $\phi \in D$,

$$
\begin{aligned}
\| U_{A}(s+h, s) & \cdot \frac{1}{h}\left[e^{B(s) h}-I\right] \phi-B(s) \phi \| \\
\leqslant & \left\|\frac{1}{h} \int_{0}^{h} U_{A}(s+h, s) e^{B(s) u} B(s) \phi-B(s) \phi d u\right\| \\
& \leqslant \sup _{0 \leqslant u \leqslant h}\left\|U_{A}(s+h, s) e^{B(s) u} B(s) \phi-B(s) \phi\right\| \\
& =\sup _{0 \leqslant u \leqslant h} \| U_{A}(s+h, s)\left(e^{B(s) u} B(s) \phi-B(s) \phi\right) \\
& \quad+U_{A}(s+h, s) B(s) \phi-B(s) \phi \| .
\end{aligned}
$$

Now for $\phi \in X, U_{A}(s+h, s) \phi \rightarrow \phi$ as $h \rightarrow 0$, uniformly in $s$ (strong continuity and compactness of $[0, t])$, and clearly $e^{B(s) u} \phi \rightarrow \phi$ uniformly in $s$ as $h \rightarrow 0$. Since $B(s) \phi$ is a compact set in $X$, and $\left\|U_{A}(s+h, s)\right\| \leqslant 1$ for all $s$ and $h$, the last term in (7) approaches zero as $h \rightarrow 0$, uniformly in $s$. (Note that $B(s) \phi$ is continuous for $s \in[0, t]$ and $\phi \in D$, for $A(s), C(s) \in \mathscr{K}$.)

Again applying uniform boundedness, we see that the convergence is uniform for $\psi \in \mathscr{K}$, as well. Finally, the last term in (5) approaches zero as $h \rightarrow 0$, for

$$
\begin{array}{r}
\left\|\frac{1}{h}\left[U_{C}(s+h, s)-I\right] \psi-C(s) \psi\right\|=\left\|\frac{1}{h} \int_{s}^{s+h} C(u) U_{C}(u, s) \psi-C(s) \psi d u\right\| \\
=\left\|\frac{1}{h} \int_{s}^{s+h} W_{C}(u, s) C(s) \psi-C(s) \psi d u\right\| \rightarrow 0 \text { as } h \rightarrow 0,
\end{array}
$$

uniformly in $s$, as already observed for $W_{A}$. This completes the proof of Theorem 1 .

Next, we determine conditions under which a partial integration formula is valid. Returning to the formal computation in (4), we see that it is now correct, provided 
the conditions of Theorem 1 are satisfied and, in addition,

(i) $\prod_{0}^{s} e^{A(u) d u}$ is invertible, with (bounded) inverse $\prod_{s}^{0} e^{A(u) d u}$, and

(ii) for each $s, \prod_{s}^{0} e^{A(u) d u} B(s) \prod_{s}^{0} e^{A(u) d u}$ (or its closure) generates a contraction semigroup.

For example, (i) will hold if, in addition, $A(s)$ generates, for each $s$, a strongly continuous group (apply the theorem on existence of product integrals to $A(s)$ and $-A(s)$ ), and (ii) will be valid provided $D_{A}$ (the common domain of the $A(s)$ 's-recall $A(\cdot) \in \mathscr{K})$ is contained in the domain of $B(s)$ for each $s$. Then it is easy to see that $\prod_{s}^{0} e^{A(u) d u} B(s) \prod_{0}^{s} e^{A(u) d u}$ is closed, densely-defined, and generates the contraction semigroup $\prod_{s}^{0} e^{A(u) d u} e^{B(s)} \prod_{0}^{s} e^{A(u) d u}$. Observing that (4) is valid under these conditions, we note that we have proved Theorem 2. In addition, we have:

Corollary 1. Formula (8) holds in the event $A(s), B(s)$ and $A(s)+B(s)$ are all skew-adjoint, satisfy the hypotheses of Theorem 1 and $D(A(s)) \subset D(B(s))$.

Proof. The proof is immediate, taking into account the remark following the definition of $\mathscr{K}$.

Corollary 2. Assume $A(s) \in \mathscr{K}$, and $B(s)$ is for each $s \in[0, t]$ a bounded dissipative operator on $X$, and $B(s)$ is strongly $C^{1}$ for $s \in[0, t]$. Then $A(s)+B(s) \in$ $\mathscr{K}$, and formula (8) holds.

Proof. Clearly $A(s)+B(s) \in \mathscr{K}$, and $\Pi_{s}^{0} e^{A(u) d u} B(s) \Pi_{0}^{s} e^{A(u) d u}$ is closed.

In conclusion, we note that our Theorems 1 and 2 may be applied in the following situations: let $X=L^{2}\left(R^{n}\right), n \geqslant 1, A(t) \equiv i \Delta$, where $\Delta$ is the selfadjoint Laplacian, and $B(t)$ is the operation of multiplication by $i V(x, t)$, where $V(x, t)$ is a realvalued measurable function on $R^{n} \times[a, b]$ and, for each $t, V(x, t) \in L^{p}\left(R^{n}\right)$, where $p \geqslant n / 2$ if $n \geqslant 5, p>2$ if $n=4$, and $p \geqslant 2$ if $n=1,2$ or 3 (cf. [4]). Additionally, assume that $V(\cdot, t) \phi(\cdot)$ is a $C^{1}$ function of $t$ for each $\phi \in D(A)$. For example, as is shown in [4, p. 103], the latter is the case provided $p$ is in the range described above, that $V(x, t)$ is $C^{1}$ in $t$ for each $x$, and $|(\partial V / \partial t)(x, t)| \leqslant \phi(x)$ for some function $\phi \in L^{p}\left(R^{n}\right)$.

More generally, we may weaken the hypotheses of Theorems 1 and 2 in an obvious way, assuming, for example, that our operator-valued functions belong to a larger class, such as $L_{s}^{1}$ (cf. [2]). Correspondingly, in the example considered above, $V(t)$ may be any selfadjoint operator that is, for each $t$, a small perturbation of $\Delta$, such that $V(t) \phi$ belongs to the appropriate class (say $L_{s}^{1}$ ) for each $\phi \in D(\Delta)$.

ACKNOWLEDGMENT. The author gratefully acknowledges helpful comments from Professors Friedman, Kato, and Melvin Heard.

\section{REFERENCES}

1. P. R. Chernoff, Note on product formulas for operator semigroups, J. Funct. Anal. 2 (1968), 238-242.

2. J. D. Dollard and C. N. Friedman, On strong product integration, J. Funct. Anal. 28 (1978), 309-354.

3. Product integration with applications to differential equations, Addison-Wesley, Massachusetts, 1979.

4. W. Faris, Product formulas for perturbations of linear propagators, J. Funct. Anal. 1 (1967), 93-108. 
5. T. Kato, (a) Integration of the equation of evolution in a Banach space, J. Math. Soc. Japan 5 (1953), 208-234; (1970), 241-258.

(b) Linear evolution equations of hyperbolic type, J. Fac. Sci. Univ. Tokyo Sect. IA Math. 17

6. P. R. Masani, The place of multiplicative integration in modern analysis, Appendix in J. D. Dollard and C. N. Friedman, Product Integration with Applications to Differential Equations, Addison-Wesley, Reading, Mass., 1979.

7. $1-9$

8. M. Reed and B. Simon, Fourier analysis, self-adjointness, Academic Press, New York, 1975.

9. K. Yosida, Functional analysis, Springer-Verlag, Berlin, 1978.

Department of Mathematics, Bryn Mawr College, Bryn Mawr, Pennsylvania 19010 